\title{
Evidence for Subcortical Plasticity after Paired Stimulation from a Wearable Device
}

\author{
${ }^{\circledR}$ Maria Germann and ${ }^{\circledR}$ Stuart N. Baker \\ Medical School, Newcastle University, Newcastle upon Tyne NE2 4HH, United Kingdom
}

Existing non-invasive stimulation protocols can generate plasticity in the motor cortex and its corticospinal projections; techniques for inducing plasticity in subcortical circuits and alternative descending pathways such as the reticulospinal tract (RST) are less well developed. One possible approach developed by this laboratory pairs electrical muscle stimulation with auditory clicks, using a wearable device to deliver stimuli during normal daily activities. In this study, we applied a variety of electrophysiological assessments to male and female healthy human volunteers during a morning and evening laboratory visit. In the intervening time $(\sim 6 \mathrm{~h})$, subjects wore the stimulation device, receiving three different protocols, in which clicks and stimulation of the biceps muscle were paired at either low or high rate, or delivered at random. Paired stimulation: (1) increased the extent of reaction time shortening by a loud sound (the StartReact effect); (2) decreased the suppression of responses to transcranial magnetic brain stimulation (TMS) following a loud sound; (3) enhanced muscle responses elicited by a TMS coil oriented to induce anterior-posterior (AP) current, but not posterior-anterior (PA) current, in the brain. These measurements have all been suggested to be sensitive to subcortical, possibly reticulospinal, activity. Changes were similar for either of the two paired stimulus rates tested, but absent after unpaired (control) stimulation. Taken together, these results suggest that pairing clicks and muscle stimulation for long periods does indeed induce plasticity in subcortical systems such as the RST.

Key words: electrical stimulation; long-term potentiation; reticulospinal; spike timing-dependent plasticity

\section{Significance Statement}

Subcortical systems such as the reticulospinal tract (RST) are important motor pathways, which can make a significant contribution to functional recovery after cortical damage such as stroke. Here, we measure changes produced after a novel noninvasive stimulation protocol, which uses a wearable device to stimulate for extended periods. We observed changes in electrophysiological measurements consistent with the induction of subcortical plasticity. This protocol may prove an important tool for enhancing motor rehabilitation, in situations where insufficient cortical tissue survives to be a plausible substrate for recovery of function.

\section{Introduction}

In primates such as humans, the corticospinal tract (CST) is the dominant descending pathway for motor control; other pathways play distinctive but lesser roles. The reticulospinal tract (RST) projects to motoneurons innervating both distal and proximal muscles in the upper limb (Davidson and Buford, 2004, 2006; Riddle et al., 2009). After CST lesions, RST connections strengthen, partially forming the substrate for recovery (Zaaimi

\footnotetext{
Received June 17, 2020; revised 0ct. 15, 2020; accepted 0ct. 21, 2020.

Author contributions: M.G. and S.N.B. designed research; M.G. performed research; M.G. analyzed data; M.G. and S.N.B. wrote the paper.

This project was supported by a Nathalie Rose Barr PhD studentship award from the International Spinal Research Trust (M.G.).

The authors declare no competing financial interests.

Correspondence should be addressed to Stuart N. Baker at stuart.baker@ncl.ac.uk.

https://doi.org/10.1523/JNEUROSCI.1554-20.2020

Copyright $\odot 2021$ the authors
}

et al., 2012). The near-absence of a rubrospinal tract in humans (Nathan and Smith, 1955; Onodera and Hicks, 2010) means that the RST may be the only major intact descending motor pathway following CST damage. It would be beneficial to develop ways of modifying RST connections to enhance functional recovery.

Neuronal connections can be strengthened if a postsynaptic neuron is activated consistently after a presynaptic input [spike timing-dependent plasticity (STDP); Markram et al., 1997]. Plasticity can be induced non-invasively by giving two appropriately-timed stimuli that converge on a common target circuit (Stefan et al., 2000; Ridding and Uy, 2003). Reticulospinal neurons receive extensive afferent (Leiras et al., 2010), auditory (Hammond, 1973; Leitner et al., 1980; Davis et al., 1982; Irvine and Jackson, 1983), and vestibular (Ladpli and Brodal, 1968; Peterson and Abzug, 1975) input. In monkey, loud auditory clicks produce robust RST activation (Fisher et al., 2012), probably via a mixture of auditory and vestibular pathways. We 
previously developed a wearable device to deliver clicks paired with electrical stimulation of the biceps muscle while a subject goes about their normal daily activities (Foysal et al., 2016). In healthy subjects, this led to plastic changes in the long-latency stretch reflex (LLSR). Potentiation or depression occurred depending on stimulus timing, consistent with STDP at the level of the brainstem. Applying this protocol in stroke survivors produced a small but significant improvement in upper limb function (Choudhury et al., 2020).

Unlike the CST, non-invasive methods to measure RST function in humans are limited. Two promising approaches use loud sound stimuli, which are of interest because they are known to elicit a startle response via the RST (Davis et al., 1982). First, loud sounds can reduce the reaction time to a visual cue (the StartReact effect). This may reflect involuntary release of a planned movement by RST (Valls-Solé et al., 1999; Rothwell, 2006; Baker and Perez, 2017), as the StartReact effect remains intact in patients with damaged CST (Honeycutt and Perreault, 2012; Nonnekes et al., 2014; Choudhury et al., 2019).

Second, loud sounds can transiently modulate motor responses to transcranial magnetic brain stimulation (TMS) and transcranial electrical stimulation (Furubayashi et al., 2000). The different effects on these two stimulus modalities points to both a cortical inhibition and subcortical facilitation; the latter may have a reticulospinal component.

TMS stimulates cells within the motor cortex both directly and indirectly (trans-synaptically), leading to multiple descending volleys to the spinal cord (Patton and Amassian, 1954; Rothwell et al., 1991). TMS over the motor cortex can activate reticular neurons trans-synaptically (Fisher et al., 2012). Although all activation involves the CST, the available evidence is that more indirect, subcortical pathways contribute to motor-evoked potentials (MEPs) elicited following late indirect (I) waves (Cirillo and Perez, 2015; Cirillo et al., 2016; Long et al., 2017). Several studies suggest that different neural elements in the cortex can be activated preferentially by changing the direction of induced currents (Werhahn et al., 1994; Sakai et al., 1997; Ni et al., 2011). Responses to anterior-posterior (AP) current may reflect more indirect cortico-muscular transmission than posterior-anterior (PA) current (Cirillo and Perez, 2015; Federico and Perez, 2017). Comparison of these responses might allow isolation of subcortical effects. As a way of controlling for spinal excitability, cervicomedullary MEPs (CMEP) can be measured, as they are thought predominantly to reflect direct effects from the CST (McNeil et al., 2013).

Here, we tested these various assessments before and after a period of paired stimulation with the wearable device. The results support an RST substrate for the induced plastic changes.

\section{Materials and Methods}

Subjects

In total, 65 healthy volunteers ( 48 females and 17 males; $18-35$ years old, right-handed by self-report) participated in the study. Four subjects took part in two experiments, one subject took part in three experiments. If participants took part in several stimulation paradigms, each session was separated by at least $7 \mathrm{~d}$. All subjects gave written informed consent to the experimental procedures, which was approved by the ethics committee of the Faculty of Medical Sciences at Newcastle University. The study was performed in accordance with the guidelines established in the Declaration of Helsinki, except that the study was not preregistered in a database.
Table 1. Summary of experiments performed

\begin{tabular}{|c|c|c|c|c|}
\hline Experiment & Intervention & $N$ & Sex $(f)$ & Assessments \\
\hline \multirow[t]{2}{*}{1} & \multirow[t]{2}{*}{ Paired } & 15 & 13 & $\begin{array}{l}\text { StartReact } \\
\text { Loud sound + TMS }\end{array}$ \\
\hline & & 12 & 10 & Loud sound + CMEF \\
\hline \multirow[t]{2}{*}{2} & \multirow[t]{2}{*}{ Double } & 14 & 9 & $\begin{array}{l}\text { StartReact } \\
\text { Loud sound + TMS }\end{array}$ \\
\hline & & 11 & 8 & Loud sound + CMEF \\
\hline \multirow[t]{2}{*}{3} & \multirow[t]{2}{*}{ Control } & 15 & 11 & $\begin{array}{l}\text { StartReact } \\
\text { Loud sound + TMS }\end{array}$ \\
\hline & & 10 & 6 & Loud sound + CMEP \\
\hline 4 & Paired & 15 & 10 & Coil orientations \\
\hline 5 & Control & 12 & 8 & Coil orientations \\
\hline
\end{tabular}

Five separate experiments (experiment 1-5) were conducted with three different device protocols (intervention: paired, double, or control). In one experiment, several assessments could be performed on the same subjects. A subset of subjects participated in assessments involving CMEPs. A few subjects participated in more than one experiment (see Materials and Methods). Experiments were conducted in the following order across the study: experiment 1, experiment 3, experiment 2, experiment 4 , experiment 5.

\section{Experimental design}

In total, five experiments were conducted, which included various assessments (Table 1). The experimental paradigm (Fig. 1A) required subjects to come to the laboratory at around 9am, where baseline assessments were conducted. They were then fitted with a wearable electronic device to deliver paired electrical and auditory stimuli, and the subject left and continued their normal daily activities. In the evening, the subject returned, the device was removed, and the assessments repeated. The order of the various assessments was randomized for a given subject, but kept the same for the morning and evening recordings in that person. The two assessment sessions were at least $6 \mathrm{~h}$ apart. For a summary of experiments, assessments and participant numbers, see Table 1.

\section{Wearable device}

The wearable device (Fig. 1B) was the same as used in our previous work (Choudhury et al., 2020). It comprised a plastic box containing an electrical stimulator and audio amplifier, powered by an internal battery which could be recharged via a standard microUSB port. The wearable device generated constant-current electrical stimulation to the right biceps muscle through surface electrodes (220-V compliance, $0.15-\mathrm{ms}$ pulse width). A knob on the device allowed adjustment of the stimulus intensity, which was set to be just below the motor threshold (defined as a visible muscle twitch). Auditory stimuli were generated by delivering a $0.1 \mathrm{~ms}$ wide, $12-\mathrm{V}$ square excitation pulse into a miniature earpiece; this produced a brief click with an intensity of $110-\mathrm{dB}$ SPL. The earpiece was placed in the left ear (contralateral to the stimulation of the right biceps). Three different interventions were tested.

For the paired stimulation group (Fig. $1 C$, top row), stimuli were delivered with an intertrial interval of $1250-1750 \mathrm{~ms}$ (chosen at random from a uniform distribution); the delay between electrical stimulation and auditory click was $10 \mathrm{~ms}$. This interstimulus interval was chosen so that the afferent volley should arrive at the brainstem just before RST cell activation by the click (as in our previous work, Foysal et al., 2016).

For the double stimulation group (Fig. $1 C$, middle row), the stimulus frequency was doubled (interval randomly chosen $575-825 \mathrm{~ms}$, uniform distribution), while the interval between biceps stimulus and click was kept at $10 \mathrm{~ms}$ as in the paired stimulation group. This allowed us to test whether increasing the stimulus rate could enhance the size of plastic changes.

For the control stimulation group (Fig. 1C, bottom row), the click and shock occurred independently at random. The time between successive clicks, and between successive biceps stimuli, followed the same distribution as in the paired stimulation group.

Participants were not told which group they were in. However, the experimenter was not blinded to the intervention.

Based on calculations given in Foysal et al. (2016) and Rosengren et al. (2010), the clicks produced by the device exposed the subjects to sound intensities of 68- $\mathrm{dB} \mathrm{L}_{\mathrm{Aeq}}$ for the paired stimulation group, and 71$\mathrm{dB} \mathrm{L}_{\mathrm{Aeq}}$ for the double stimulation group (assuming device usage for at 
A

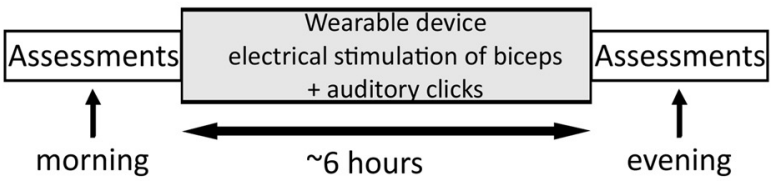

B

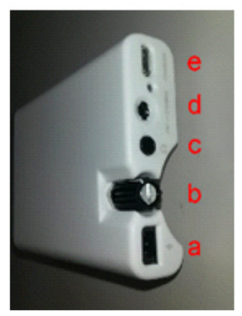

C

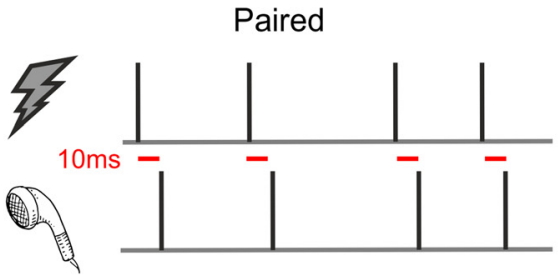

Double

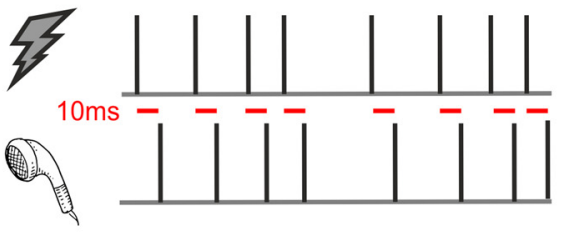

Control

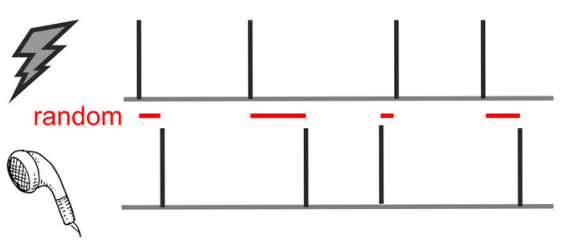

Figure 1. Wearable device and schematic diagram showing the different stimulus conditions. $\boldsymbol{A}$, General experimental protocol. $\boldsymbol{B}$, Photograph of the wearable device: $(\boldsymbol{a})$ stimulus output port; (b) stimulus intensity adjustment; (c) audio output; (d) on/charge switch; (e) microUSB charge connection. $C$, The three different stimulus conditions implemented by the wearable device. Top, Paired stimulation group, with 10-ms interstimulus interval between clicks and biceps electrical stimuli. Middle, Double stimulation group, with 10-ms interstimulus interval, but double the stimulation frequency as the other groups. Bottom, Unpaired control stimulation group, with random interstimulus intervals.

most $8 \mathrm{~h}$ ). This is well below accepted hearing safety limits for noise exposure of $85-\mathrm{dB} \mathrm{L}_{\text {Aeq }}$ (The Stationery Office, 2005); the device could potentially be used every day at these levels without concern.

\section{Electromyography (EMG) recordings}

EMG was recorded through surface electrodes (disposable Kendall H59P Electrodes, Covidien) secured on the skin over the belly of the biceps or the right first dorsal interosseous (1DI) muscle. Because the electrodes over the right biceps muscle were used for both EMG recording and for wearable stimulation, they were kept in place for the whole day, ensuring consistency between the morning and evening sessions. For the right $1 \mathrm{DI}$ and the left biceps, the location was marked after the morning session with a UV fluorescent marker pen, allowing fresh electrodes to be replaced in the same location in the evening. EMG signals were amplified and filtered $(30-2000 \mathrm{~Hz}$, gain 1000) with a bioamplifier (D360 Amplifier, Digitimer), digitized at a sampling rate of $5 \mathrm{kHz}$ (CED Micro 1401 with Spike2 software, Cambridge Electronic Design) and stored on a computer for off-line analysis. Where necessary for a given assessment (see below), the level of biceps EMG activity was displayed continuously on a computer screen via series of colored bars, allowing subjects to maintain a constant isometric contraction.

TMS

Transcranial magnetic stimuli were applied using a figure-of-eight coil through a Magstim $200^{2}$ magnetic stimulator (Magstim) with a monophasic current waveform. We determined the optimal position for eliciting a MEP in the biceps muscle (hotspot) by moving the coil, with the handle pointing backward and $45^{\circ}$ from the midline, in small steps along the arm representation of M1. The hotspot was defined as the region where the largest MEP in the biceps muscle could be evoked with the minimum intensity (Rothwell et al., 1999). Unless stated otherwise, the magnetic coil was held in this orientation over the left hemisphere for subsequent measurements, to induce currents in the brain that flowed perpendicular to the presumed line of the central sulcus in a PA direction. Active motor threshold (AMT) was defined as the stimulator intensity sufficient to elicit a MEP with amplitude $>200 \mu \mathrm{V}$ in at least five out of 10 consecutive stimuli, in the biceps contralateral to the stimulus with an active contraction of 10-20\% maximal voluntary contraction (MVC).

A Polaris Vicra camera (Northern Digital Inc.) tracked both coil and head position, allowing the site of stimulation to be marked using the Brainsight neuronavigation system (Rogue Research Inc.). This ensured a stable coil location throughout the experiment, and allowed us to return to the same site in the evening as had been used earlier in the day.

Cervicomedullary junction stimulation

CMEPs were elicited via electrical stimulation of the CST at brainstem level. Adhesive surface electrodes (Neuroline 720 00-S/25, Ambu) were fixed to the skin over the mastoid processes and current was passed between them (0.1 ms duration, $80-200 \mathrm{~mA}$; model DS7AH, Digitimer Ltd) with the cathode on the left side. The stimulation intensity was adjusted to elicit a CMEP amplitude of $1 \mathrm{mV}$ with an active contraction of $10-20 \%$ MVC.

\section{Assessment: StartReact}

StartReact was examined using a previously tested paradigm (Baker and Perez, 2017) which measures reaction time from EMG in response to a visual cue [visual reaction time (VRT)], a visual plus quiet auditory cue [visual-auditory reaction time (VART)], and a visual plus loud auditory cue [visual-startle reaction time (VSRT)]. The acceleration of reaction time between VART and VSRT is believed to involve the rapid involuntary release of the preprepared movement, and to reflect the action of the RST (Valls-Solé et al., 1999; Carlsen et al., 2004; Rothwell, 2006; Baker and Perez, 2017; Smith et al., 2019). Some previous studies on StartReact have measured startle responses from the sternocleidomastoid (SCM) muscle, and excluded reaction times measured when this muscle was not activated (Valls-Solé et al., 1999; Carlsen et al., 2004; Honeycutt et al., 2013). We and others have previously found that SCM activity is unreliable (Honeycutt et al., 2013; Baker and Perez, 2017; Dean and Baker, 2017), and can be generated both in response to startling and non-startling cues. In this study, we therefore simply included all reaction times measured from the relevant cue, as in our previous publications (Baker and Perez, 2017; Dean and Baker, 2017; Choudhury et al., 2019).

Subjects sat with the right forearm supinated and placed on the table top, and the hand loosely placed around a horizontal handle. A green light-emitting diode (LED) was located $\sim 1 \mathrm{~m}$ in front of the subject. Subjects were instructed to grip the handle and push upwards as quickly as possible after the LED illuminated. EMG was recorded from both the $1 \mathrm{DI}$ and biceps muscles, and reaction time measured as the time from cue to onset of the EMG burst. Three types of trial were randomly interleaved (20 repeats per condition; intertrial interval 5-6 s; Fig. 2A): LED illumination alone (VRT), LED paired with a quiet sound $(80 \mathrm{~dB}$, 
A

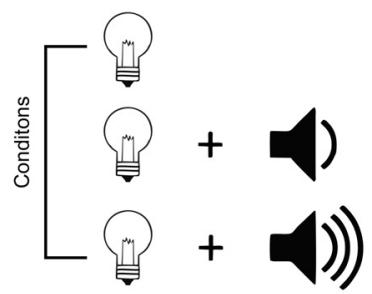

C

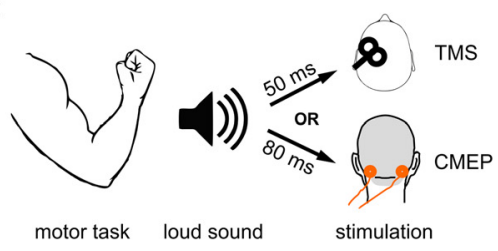

E

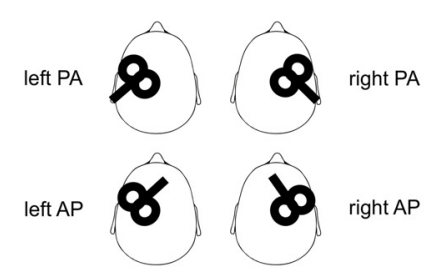

B

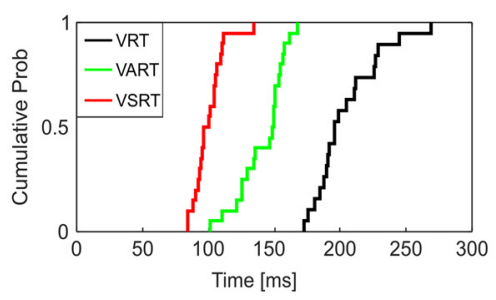

D

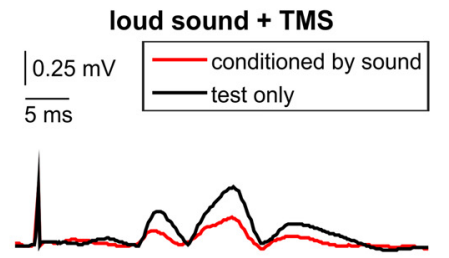

$\mathbf{F}$

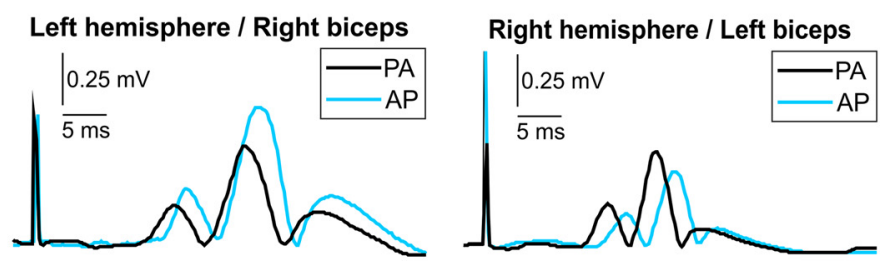

Figure 2. Experimental setup and single subject example for the different assessments. $\boldsymbol{A}$, The three conditions tested for the StartReact assessment; VRT, VART, and VSRT. B, Cumulative distribution of EMG onset times for all trials of a single subject at baseline. $C$, Setup for the two loud sound assessments. For the loud sound + TMS assessment, sound was delivered $50 \mathrm{~ms}$ before TMS pulse. For the loud sound + CMEP assessment, sound was delivered $80 \mathrm{~ms}$ before stimulation. $\boldsymbol{D}$, Average rectified EMG traces at baseline of a single subject for conditioned (red) and unconditioned (black) MEPs for the two assessments. $\boldsymbol{E}$, Setup for the assessment of MEPs using different coil orientations and lateralities. Stimulation over the left hemisphere elicited MEPs in the right biceps, which received wearable device stimulation; right hemisphere stimulation elicited MEPs in the left biceps, which did not receive wearable device stimulation. $\boldsymbol{F}$, Average rectified EMG traces at baseline of a single subject for MEPs elicited with PA (black) or AP (blue) coil orientation at baseline from each hemisphere.

$500 \mathrm{~Hz}, 50 \mathrm{~ms}$, VART), LED paired with a loud sound $(500 \mathrm{~Hz}, 50 \mathrm{~ms}$, $120 \mathrm{~dB}$, VSRT). Subjects were initially presented with five consecutive loud sounds, without performing the task, to allow familiarization.

Data were analyzed trial-by-trial using a custom MATLAB program which identified the reaction time as the point where the rectified EMG exceeded the mean +7 SD of the baseline measured 0-200 ms before the stimulus. Every trial was inspected visually, and erroneous activity onset times (caused, for example, by electrical noise artefacts) were manually corrected. This yielded a reaction time distribution for each condition (Fig. 2B).

\section{Assessment: loud sound with MEP or CMEP}

A paradigm similar to previous studies (Furubayashi et al., 2000; Tazoe and Perez, 2017) was used (Fig. 2C). Loud (500 Hz, 120-dB SPL) sound stimuli were given $50 \mathrm{~ms}$ before a TMS pulse over M1; this elicits a smaller MEP than the same TMS pulse given alone, which is believed to reflect cortical inhibitory processes. Similar sound stimuli were also given $80 \mathrm{~ms}$ before electrical stimulation of the cervicomedullary junction; this causes a facilitation of the CMEP compared with that elicited by electrical stimulation alone, which likely arises from subcortical circuits. The two intervals used were selected as these gave the largest suppression and facilitation respectively in a previous detailed examination of these effects (Furubayashi et al., 2000). Sounds were given through two audio speakers located on a table $\sim 1 \mathrm{~m}$ in front of the subject. The sound ended at the time of the test stimulus. To avoid stimulus predictability, the intertrial interval was chosen randomly between 20.5 and $23 \mathrm{~s}$ (uniform distribution). At the beginning of the study, five consecutive loud sounds were presented to habituate the startle reflex. During testing, subjects sat with the arm resting on their leg under a table top, and pushed up against the table at 10-20\% of MVC. The level of biceps EMG activity was displayed continuously on a computer screen via a series of colored bars, allowing subjects to maintain a constant isometric contraction, which was kept the same across sessions for that subject. The test stimulus intensity was adjusted to elicit a peak-to-peak MEP of $1 \mathrm{mV}$ in the right biceps muscle. Ten test MEPs and 10 conditioned MEPs were measured in each condition, in randomized order. As expected from previous work, at the tested interval of 50-ms MEPs were typically suppressed, whereas with the 80 -ms interval CMEPs were facilitated (see examples in Fig. 2D). Some of the CMEP traces showed a small early component (Fig. 2D, small "shoulder" at the onset of the CMEP responses), with an onset latency which may be consistent with direct stimulation of the spinal motor roots (McNeil et al., 2013). However, this was small compared with the later component which arose from corticospinal stimulation and trans-synaptic activation of motoneurons. A direct root response would not be expected to modulate (as is the case for the response shown in Fig. 2D), so that the presence of this small component would not have materially affected our results.

CMEPs can be uncomfortable for the subject, and several participants withdrew from this part of the study; numbers participating in both CMEP and MEP assessments are given in Table 1.

\section{Assessment: MEPs with different coil orientations}

By holding a figure-of-eight TMS coil in different orientations, activation can be biased toward different indirect I waves (Sakai et al., 1997; Ziemann and Rothwell, 2000). With the coil tangential to the scalp, at an angle of $45^{\circ}$ to the midline with the handle pointing laterally and posteriorly, a PA current is induced in the brain, which mainly recruits early I waves. By contrast, in the reverse orientation (handle pointing medial and anterior; AP-induced current), predominantly late I waves are recruited. Here, we measured MEPs with different coil orientations to compare changes in direct versus indirect corticospinal pathways. We assessed changes bilaterally, as the cortico-reticulospinal system is known to be bilaterally organized: RST axons project to both sides of the spinal cord (Peterson et al., 1975; Davidson and Buford, 2006; Davidson et al., 2007), and cells within the reticular formation can be stimulated by TMS to M1 over either ipsilateral or contralateral hemispheres (Fisher et al., 2012).

MEPs in the contralateral biceps muscle were measured from both hemispheres in two different coil orientations (Fig. 2E). As for the measurements of MEPs described above, the figure-of-eight coil was first held 
A

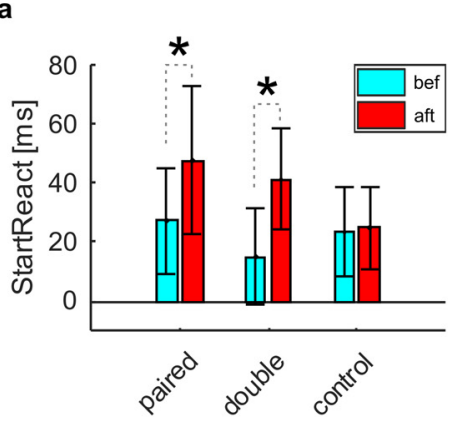

B

a

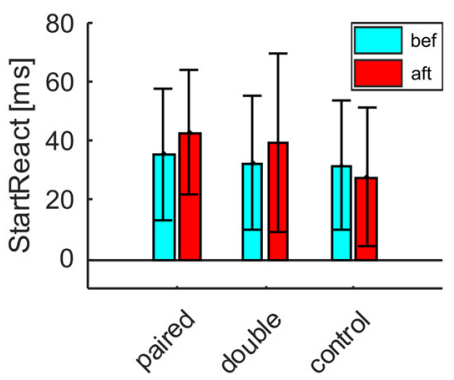

Biceps StartReact

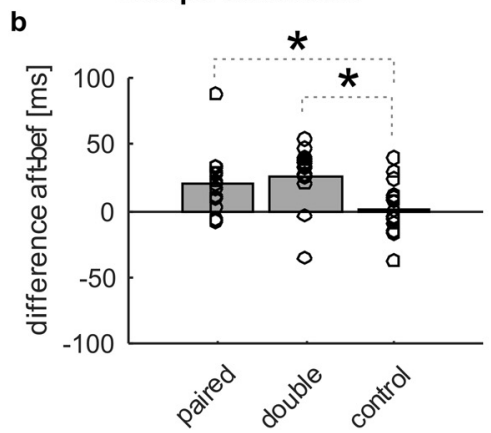

1DI StartReact

b

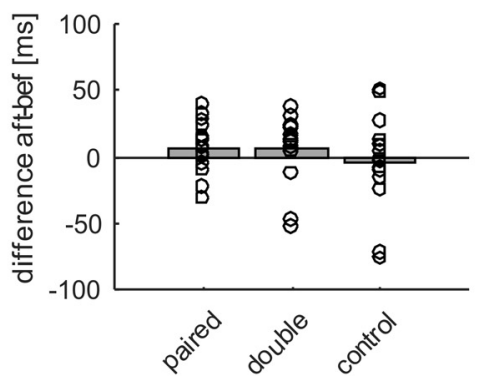

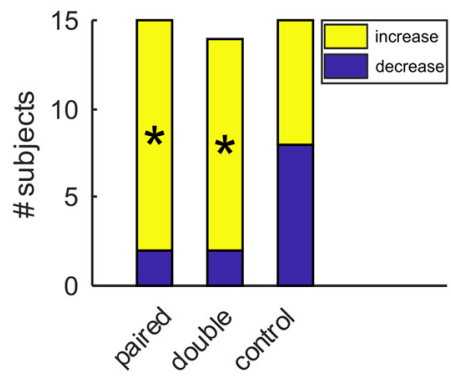

c

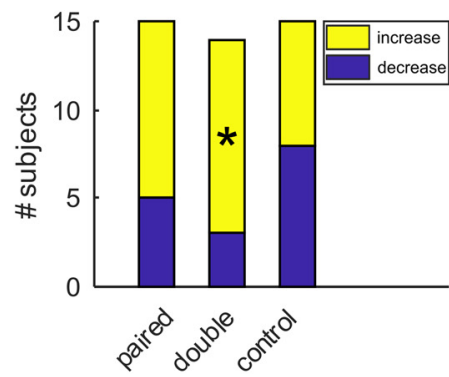

Figure 3. Group results for the StartReact assessment. $\boldsymbol{A}$, Results for the biceps muscle (paired $n=15$; double $n=14$; control $n=15$ ). $\boldsymbol{a}$, StartReact effect (difference between VART and VSRT) before (cyan) and after (red) wearable device stimulation. Colored bars represent group means; error bars indicate SDs. Asterisks indicate significant differences. $\boldsymbol{b}$, Difference in StartReact effect between before and after wearable device stimulation. A positive difference indicates a more pronounced StartReact effect after wearable device stimulation. Bars represent group means; circles show single subject values. c, Number of subjects showing an increase (yellow) or decrease (blue) in StartReact after wearable device stimulation. Asterisks indicate proportions significantly different from the $50 \%$ expected by chance. $\boldsymbol{B}$, Results for the $1 \mathrm{DI}$ muscle, in the same format as $\boldsymbol{A}$.

to induce a PA current in the brain. The optimal scalp position was located with this orientation, and marked in the Brainsight neuronavigation software. This site was then used for stimulation to induce an AP current in the brain, by rotating the coil so that the handle faced anterior and medial, as the current direction does not significantly influence the position of the hotspot (Sakai et al., 1997; Arai et al., 2005). Within each subject, we randomized the order of hemispheres tested (left vs right), and for each side the order of coil orientations (PA vs AP); the same order was used for the morning and evening assessments. Stimulus intensity was set to $1.1 \times$ AMT (four separate thresholds were measured, one for each hemisphere and coil orientation), while subjects contracted to $10 \%$ MVC. The level of biceps EMG activity was displayed continuously on a computer screen via a series of colored bars. 20 MEPs were recorded (5-s interstimulus interval) while subjects maintained a constant isometric contraction of $10 \%$ MVC. As expected from previous work, the MEP onset latency was slightly later for AP versus PA orientation (see example traces from one subject in Fig. $2 F$ ).

\section{Data analysis}

Data were analyzed using custom scripts written in the MATLAB environment (R2017a, MathWorks). Statistical tests were performed using MATLAB and IBM SPSS Statistics for Windows, version 24 (IBM Corp.).

EMG traces were full-wave rectified and then averaged. MEP amplitude was measured as the area under the curve of this average. Where MEPs were conditioned by loud sound, the amplitude of the conditioned MEP was expressed as a percentage of the unconditioned MEP. The StartReact effect was measured as the difference between the mean VART and VSRT.

For each experiment, individual paired $t$ tests were used to compare the measurements after wearable device stimulation to the measurements at baseline. In order to compare stimulation groups, one-way ANOVAs were performed on the differences (after-before wearable device intervention) for each measurement. Independent $t$ tests were used to compare groups post hoc. Where necessary, the procedure introduced by Benjamini and Hochberg (1995) to correct for multiple comparisons was used. Cohen's $d$ was used to measure effect size. Monte Carlo methods were performed to determine whether the number of subjects showing a certain change were more than expected by chance based on a binomial distribution.

For the assessment which conditioned MEPs with loud sound, a one sample $t$ test was performed for each group to compare the normalized conditioned MEP amplitude against 100\%, to evaluate the effect of conditioning at baseline.

The significance level was set at $p<0.05$. Descriptive statistics are presented as mean $\pm \mathrm{SD}$.

\section{Results}

\section{StartReact}

Figure 3 presents results for the StartReact assessment, for both the biceps and 1DI muscles.

In biceps, there was a significantly larger StartReact effect after paired $(p=0.004)$ and double $(p<0.001)$ stimulation, but no difference after unpaired $(p=0.760)$ stimulation (Fig. 3Aa). The difference in the StartReact effect after wearable device stimulation compared with baseline (Fig. $3 A b$ ) was significantly different for the three stimulation groups $\left(F_{(2,41)}=5.233, p=0.009\right.$, partial $\left.\eta^{2}=0.203\right)$. Post hoc $t$ tests revealed that the paired and the double stimulation groups showed a significantly bigger difference in the StartReact effect compared with the control group (paired group $t_{(28)}=-2.445, p=0.021, d=-0.893$; double group $t_{(27)}=$ $-3.160, p=0.004, d=-1.175)$ but that there was no difference between the paired and the double stimulation group $\left(t_{(27)}=\right.$ $0.673, p=0.507)$.

The analysis presented so far shows average effects across all subjects tested, which is appropriate for statistical analysis. However, it is known that many plasticity protocols have variable 
$\mathbf{A}_{\mathrm{a}}$

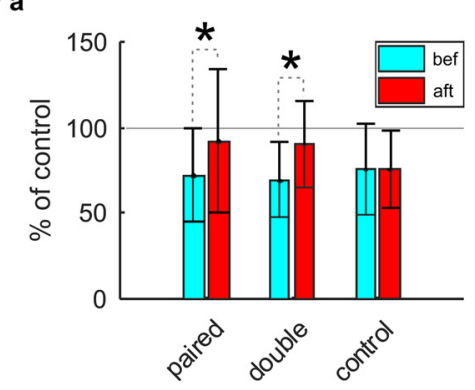

B

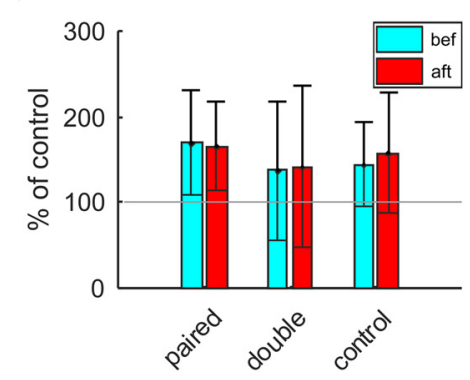

b

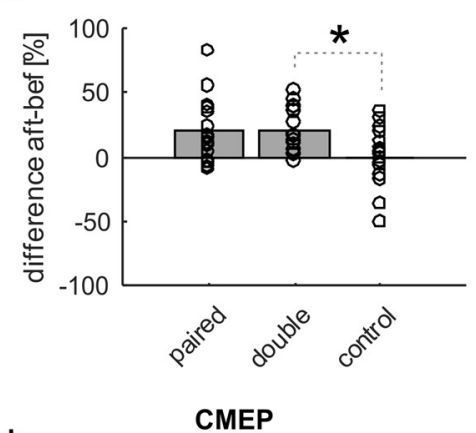

b

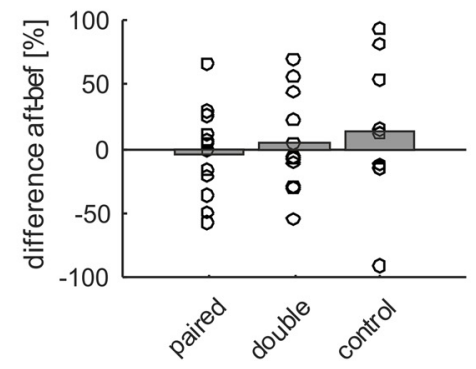

c

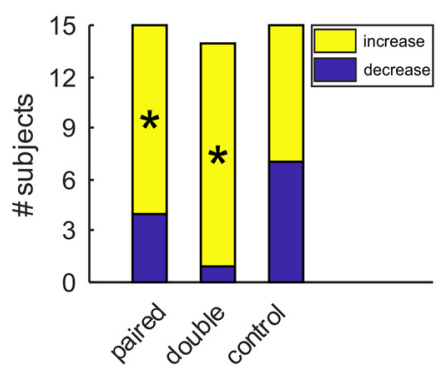

C

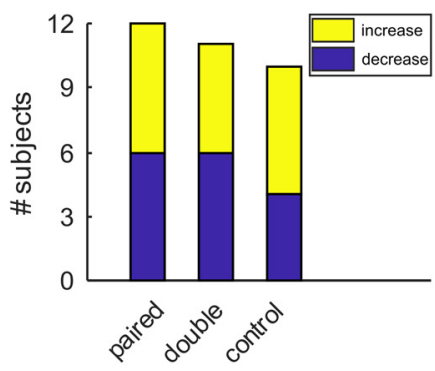

Figure 4. Group results for the conditioning MEPs with loud sounds. $\boldsymbol{A}$, Group results for the loud sound + TMS assessment at 50-ms interval (paired $n=15$; double $n=14$; control $n=15$ ). $\boldsymbol{a}$, Conditioned MEP amplitude normalized to unconditioned amplitude, before (cyan) and after (red) wearable device stimulation for the three different stimulation groups. Colored bars represent group means; error bars indicate SDs across subjects. Asterisks indicate significant differences. $\boldsymbol{b}$, Difference in normalized MEP amplitude between before and after wearable device stimulation. A positive difference indicates less suppression of the conditioned MEP after wearable device stimulation. Bars represent group means; circles show single subject values. $\boldsymbol{c}$, Number of subjects showing an increase (yellow) or decrease (blue) in conditioned MEP amplitude after wearable device stimulation. Asterisks indicate proportions significantly different from the $50 \%$ expected by chance. $\boldsymbol{B}$, Group results for the loud sound + CMEPs assessment at 80-ms interval (paired $n=12$; double $n=11$; control $n=10$ ). Layout is the same as for $\boldsymbol{A}$.

effects across subjects (Cheeran et al., 2008; Wiethoff et al., 2014). Similar to our previous publications (Foysal et al., 2016; Foysal and Baker, 2019), Figure 3Ac presents the fraction of subjects in which the StartReact effect increased or decreased, with the aim of providing information about the consistency of the effect. There was an increase in the StartReact effect in more subjects than expected by chance for the paired $(13 / 15 ; p<0.001$, binomial test) and the double $(12 / 14 ; p=0.001)$ but not the control $(7 / 15 ; p=0.992)$ groups (Fig. $3 A c)$.

To respond to the cues, participants had to tighten their grip around the handle and push up against it. It was therefore possible to record a response in the 1DI muscle, as well as in biceps. Figure $3 B$ shows results for StartReact measured in this muscle, which is of interest as it did not receive any wearable device stimulation. There was no significant increase in the StartReact effect in any of the groups after wearable device stimulation (paired group $p=0.179$; double group $p=0.364$; control group $p=$ 0.681 ; Fig. $3 \mathrm{Ba}$ ), and no effect of stimulation group on StartReact difference $\left(F_{(2,41)}=0.729, p=0.489\right.$, partial $\eta^{2}=0.034$; Fig. $\left.3 B b\right)$. However, a majority of subjects did show an increased StartReact effect after paired (10/15) and double (11/14) stimulation, but not unpaired (7/15) stimulation (Fig. 3Bc). This was significantly higher than chance only for the double stimulation group $(p=0.012)$, but not for the paired $(p=0.110)$ or the control $(p=0.598)$ group.

\section{MEPs conditioned by loud sounds}

Figure $4 A$ shows the results when MEPs elicited by TMS were conditioned by a loud sound beginning $50 \mathrm{~ms}$ before the magnetic stimulus. As expected from previous studies (Furubayashi et al., 2000; Tazoe and Perez, 2017), at baseline MEPs were significantly suppressed in all three groups (cyan bars below gray
$100 \%$ line in Fig. $4 A a$; one sample $t$ test paired group $p=0.001$; double group $p<0.001$; control group $p=0.004$ ). For both the paired and the double stimulation group, conditioned MEPs were significantly less suppressed after wearable device stimulation (red bars compared with cyan bars; paired group $p=0.009$; double group $p<0.001)$. On the other hand, the control stimulation group, which received unpaired stimuli, did not show any significant difference after wearing the device $(p=0.993)$.

Figure $4 A b$ plots the difference in MEP amplitude after compared with before the wearable device intervention for each group; a positive difference here indicates less suppression of the conditioned MEP after wearable device stimulation. A one-way ANOVA showed that the three stimulation groups differed significantly $\left(F_{(2,41)}=4.044, p=0.025\right.$, partial $\left.\eta^{2}=0.165\right)$. Post hoc $t$ tests confirmed there was a significantly bigger difference in the double stimulation group compared with the control stimulation group $\left(t_{(27)}=2.708, p=0.012, d=1.006\right)$. There was also a larger difference in the paired stimulation group compared with the control; however, this just failed to reach significance $\left(t_{(28)}=2.227, p=0.034, \mathrm{~d}=0.815\right.$; threshold for significance $p<0.033$ using Benjamini-Hochberg correction for multiple comparisons). There was no significant difference between the paired and the double stimulation group $\left(t_{(27)}=\right.$ $-0.128, p=0.899)$. Significantly more participants showed MEP increases than the $50 \%$ expected by chance after paired $(11 / 15 ; p=0.035$, binomial test $)$ and double $(13 / 14 ; p<0.001)$ stimulation, but not after unpaired stimulation $(8 / 15 ; p=$ 0.604; Fig. 4Ac).

Figure $4 B$ presents results for conditioning of CMEPs with loud sounds, in the same format as Figure $4 A$. CMEPs were facilitated by a loud sound at $80-\mathrm{ms}$ interval at baseline in all groups (cyan bars above gray $100 \%$ line; Fig. 4Ba). This facilitation was significant for the paired and control groups, but not 
A

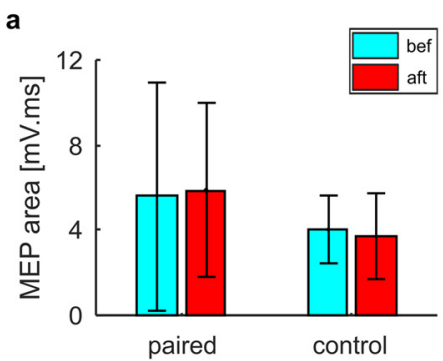

B

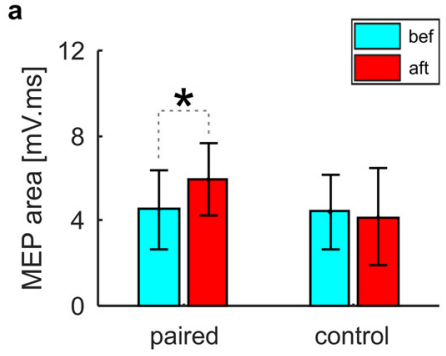

C

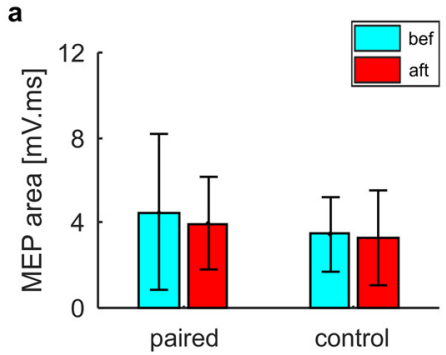

D

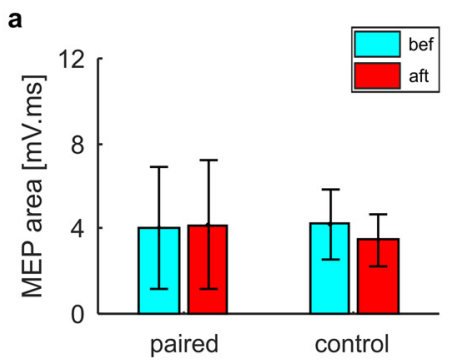

left | PA

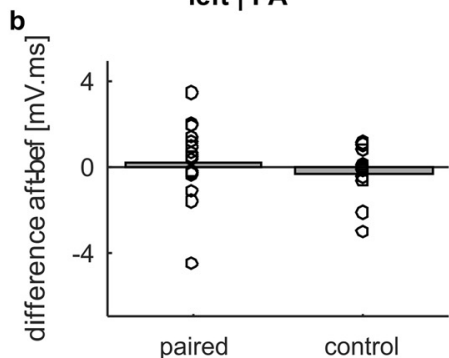

left | AP

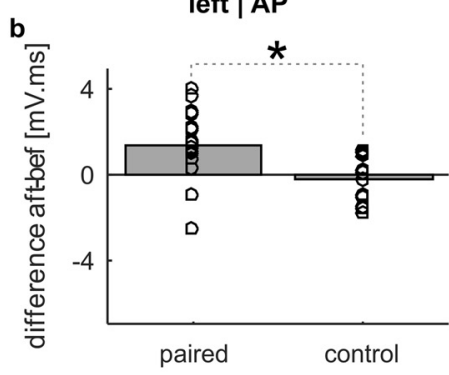

right | PA

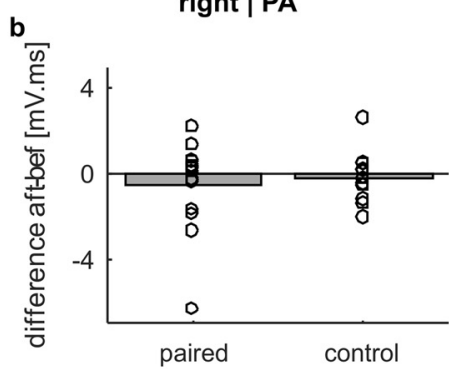

right | AP

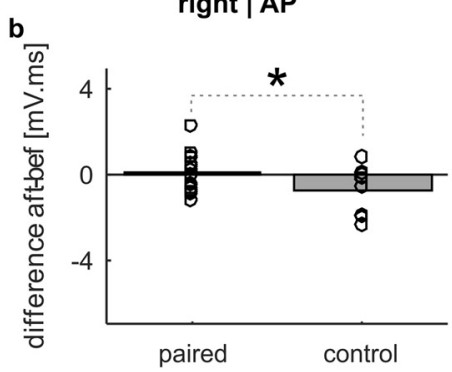

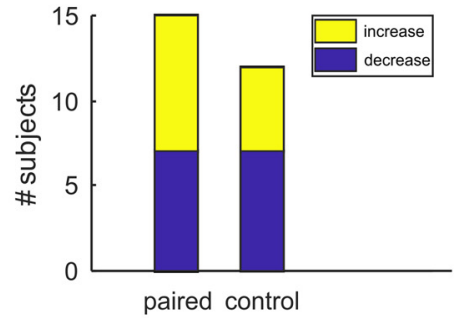

c
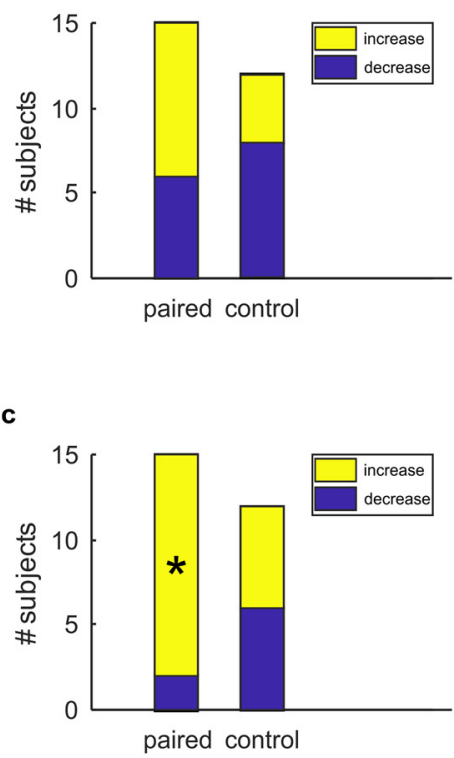

c

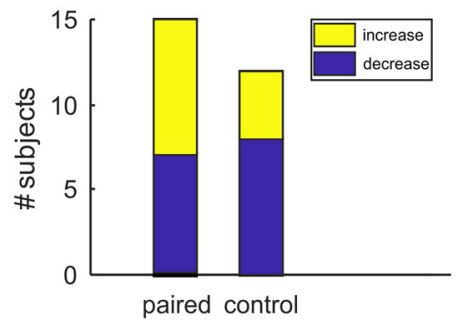

Figure 5. Group results for the coil orientations assessment. $\boldsymbol{A}$, Results when holding the coil in PA orientation and stimulating over the left motor cortex. $\boldsymbol{a}$, MEP amplitude before (cyan) and after (red) wearable device stimulation for the two different stimulation groups (paired $n=15$; control $n=12$ ). Colored bars represent group means; error bars indicate SDs. Asterisks indicate significant differences. $\boldsymbol{b}$, Difference in MEP amplitudes between before and after wearable device stimulation. A positive difference indicates an increase in MEP size after wearable device stimulation. Bars represent group means; circles show single subject values. $c$, Number of subjects showing an increase (yellow) or decrease (blue) in MEP amplitude after wearable device stimulation. Asterisks indicate proportions significantly different from the $50 \%$ expected by chance. $\boldsymbol{B}$, Results for an AP coil orientation stimulating over the left motor cortex. $\boldsymbol{C}$, Results for a PA coil orientation stimulating over the right motor cortex. $\boldsymbol{D}$, Results for an AP coil orientation stimulating over the right motor cortex.

the double stimulation group $(p=0.024, p=0.018$, and $p=0.157$, respectively). There was no significant difference after wearable device stimulation compared with baseline in any of the groups (paired group $p=0.690$; double group $p=0.718$; control group $p=0.476$; Fig. $4 \mathrm{Ba})$ and the groups did not differ significantly from each other $\left(F_{(2,30)}=0.423, p=0.659\right.$, partial $\eta^{2}=0.027$; Fig. $4 B b)$. The number of participants showing a positive or negative difference was approximately equal in all groups and not significantly higher than the $50 \%$ expected by chance for any group (paired group $p=0.783$; double group $p=0.990$; control group $p=0.336$; Fig. $4 B c$ ). Overall, our results indicate that there was no change in conditioned CMEP amplitude after any of the wearable device stimulation paradigms.

\section{MEPs measured bilaterally with different coil orientations}

Figure 5 shows the group results for the four combinations of coil orientation and laterality tested.

When the coil was held in the PA orientation over the left hemisphere (contralateral to the arm stimulated by the wearable device), there was no change in MEP size in the contralateral biceps after wearable device stimulation (paired group $p=0.572$; control group $p=0.724$; Fig. 5Aa) and changes for the two 
stimulation groups were not significantly different $\left(F_{(1,25)}=\right.$ $0.909, p=0.350$, partial $\left(\eta^{2}\right)=0.035$; Fig. $\left.5 A b\right)$. The number of subjects with increased MEPs was no different from the distribution expected by chance (paired group 9/15, $p=0.307$; control group 4/12, $p=0.152$; Fig. $5 A c$ ).

In marked contrast, when the coil was held in the AP position, there was a significant increase in MEP amplitude after paired $(p=0.006)$, but not after control $(p=0.925)$ stimulation (Fig. $5 \mathrm{Ba}$ ). The difference in MEP amplitude after wearable device stimulation was significantly greater in the paired group $\left(F_{(1,25)}=8.585, p=0.007\right.$, partial $\left(\eta^{2}\right)=0.256$; Fig. $\left.5 B b\right)$. Significantly more subjects showed an increase in MEP amplitude than expected by chance after paired $(13 / 15 ; p=0.006)$ but not after control $(6 / 12 ; p=0.765)$ stimulation (Fig. $5 B c$ ).

Figure $5 C$ presents results from PA TMS applied over the right hemisphere (ipsilateral to the stimulated arm) and MEPs measured in the contralateral biceps, which had not received wearable device stimulation. There was no change in MEP size in the left biceps in either group (paired group $p=0.338$; control group $p=0.596$; Fig. $5 \mathrm{Ca}$ ) and the two stimulation groups did not differ from each other $\left(F_{(1,25)}=0.263, p=0.612\right.$, partial $\left(\eta^{2}\right)$ $=0.010$; Fig. $5 C b$ ). The number of participants showing an increase or decrease in MEP amplitude was approximately equal in both groups (paired group $8 / 15, p=0.293$; control group 5/12, $p=0.370$; Fig. $5 C c$ ).

When TMS was applied in the AP orientation over the right hemisphere (Fig. 5D), there was no change in MEP size for the paired group $(p=0.647)$, or after unpaired stimulation (control group $p=0.029$; threshold for significance $p<0.025$ using Benjamini-Hochberg correction for multiple comparisons; Fig. 5Da). A one-way ANOVA showed that MEP amplitude decreased slightly but significantly more after unpaired stimulation compared with after paired stimulation $\left(F_{(1,25)}=5.061\right.$, $p=0.034$, partial $\left(\eta^{2}\right)=0.168$; Fig. $\left.5 D b\right)$. However, the number of subjects showing a decrease in MEP size was not significantly higher than chance for either group (paired $7 / 15$, $p=0.299$; control 8/12, $p=0.148$; Fig. $5 D c$ ).

\section{Discussion}

\section{Converging evidence for subcortical plasticity}

After wearable device paired stimulation at either of the two rates tested, the StartReact effect increased. Importantly, this change only occurred in the biceps muscle which had been stimulated by the wearable device; there was no change in the unstimulated 1DI muscle. This appears to follow the plasticity principle of specificity (Barrionuevo and Brown, 1983). StartReact reflects the involuntary release of a planned movement, and several studies suggest that the size of this effect is a measure of RST outflow (Valls-Solé et al., 1999; Carlsen et al., 2004; Rothwell, 2006; Baker and Perez, 2017; Smith et al., 2019). The changes seen in StartReact are thus consistent with a strengthening of RST output to the biceps motor nucleus after wearable device stimulation.

A single TMS pulse evokes multiple descending volleys to the spinal cord: an initial direct (D) followed by subsequent I waves (Patton and Amassian, 1954; Rosenthal et al., 1967; Stoney et al., 1968; Jankowska et al., 1975). I waves are further classified as early (I1, I2) and late (I3, etc.; Patton and Amassian, 1954; Rothwell et al., 1991). TMS can preferentially recruit different descending waves in humans by changing the direction of the current induced in the brain (Sakai et al., 1997; Ziemann and Rothwell, 2000). PA-directed current predominantly recruits early, whereas AP current predominantly recruits late I waves (Day et al., 1989; Sakai et al., 1997; Di Lazzaro et al., 2001; Ni et al., 2011). Several studies in humans suggest that subcortical pathways contribute to muscle responses to late I waves (Cirillo and Perez, 2015; Cirillo et al., 2016; Long et al., 2017). In monkey, repetitive stimulation of the CST generates a supernumerary extra volley in the RST (Fisher et al., 2015); the repetitive corticospinal activation produced by successive I waves could have a similar effect.

We considered first MEPs elicited in the biceps muscle which had received wearable device stimulation, after TMS over the contralateral hemisphere. After wearable device stimulation, the MEP amplitude following TMS with PA oriented current was unchanged. Such MEPs are likely to originate from early I waves, and activate motoneurons predominantly via monosynaptic corticomotoneuronal connections. This result therefore argues that there were no changes in either the strength of corticospinal connections, or changes in cortical excitability. By contrast, MEP amplitude after TMS using AP oriented current was significantly increased following paired stimulation. This could arise from selective changes in intracortical circuits generating late I waves, but equally could occur following subcortical plasticity, for example increased efficacy of cortico-reticular or reticulospinal connections. This would lead to enhanced activation of the biceps motor nucleus.

The RST makes bilateral projections to the spinal cord (Peterson et al., 1975; Davidson and Buford, 2006; Davidson et al., 2007), and the reticular nuclei receive inputs from both contralateral and ipsilateral hemispheres (Fregosi et al., 2017; Darling et al., 2018; Fisher et al., 2021). We were therefore interested to assess changes contralateral to the arm which had received wearable device stimulation. We found no significant changes in MEPs following paired stimulation for either of the tested coil orientations. Control stimulation may have caused a small decrease in MEPs from the right hemisphere relative to paired stimulation, although this effect was weak. It may represent a slight non-specific effect of the stimulation. In our previous study, delivering biceps stimulation after clicks induced a reduction in the $\mathrm{R} 1$ component of the stretch reflex, which is likely to reflect mainly spinal processes (Foysal et al., 2016).

When testing the effect of conditioning MEPs or CMEPs with loud sound, multiple overlapping processes are likely to contribute to the net response facilitation or suppression which is observed. Loud sounds facilitate the $\mathrm{H}$ reflex at intervals larger than $50 \mathrm{~ms}$ (Rossignol and Jones, 1976; Rudell and Eberle, 1985; Nakashima et al., 1994; Delwaide and Schepens, 1995), indicating an increase in motoneuronal excitability. The suppression of TMS-evoked MEPs seen at the 50-ms interval (Furubayashi et al., 2000; Kühn et al., 2004; Tazoe and Perez, 2017) must therefore reflect a cortical suppression superimposed on a smaller spinal facilitation. The cortical suppression most likely results from stimulation of the reticular formation by the loud sound (Hammond, 1973; Leitner et al., 1980; Davis et al., 1982; Fisher et al., 2012), and activation of cortical interneurons via reticulothalamic projections (Pare et al., 1988; Steriade et al., 1988). Stimulation at the cervicomedullary junction activates the CST at the brainstem level (Ugawa et al., 1991), and hence the size of the descending volley should be unaffected by cortical excitability. In the biceps muscle, this stimulation can evoke responses consistent with both monosynaptic (Petersen et al., 2002) and oligosynaptic transmission (Nakajima et al., 2017). At an 80-ms interval, although CMEPs and $\mathrm{H}$ reflexes are facilitated, MEPs are not (Furubayashi et al., 2000; Tazoe and Perez, 2017), 
suggesting that the cortical suppression persists at this interval and is now equally balanced by spinal facilitation.

In this study, we found that the MEP suppression at the 50$\mathrm{ms}$ interval was reduced after paired wearable device stimulation, but that the CMEP facilitation at $80 \mathrm{~ms}$ was unchanged. This may reflect a decrease in cortical inhibition elicited by the loud sound. It is also possible that the reduced net suppression arose from an increase in spinal facilitation, which might arise from subcortical pathways, although then we might have expected to see an increase in the CMEP facilitation at $80 \mathrm{~ms}$. Our results have strong similarity to the work of Tazoe and Perez (2017), who measured these effects in the contracting 1DI muscle while subjects performed different types of grasp. During a power grip MEP suppression was smaller, and CMEP facilitation the same as during an isolated index finger abduction. These authors argued that the reduced suppression was indicative of a smaller cortical contribution to the motoneuron drive during power grip, whereas the similarity of CMEP facilitation indicated no change in corticomotoneuronal synaptic efficacy or motoneuron excitability. Our results may similarly indicate a relative shift away from corticospinal drive to activate biceps after wearable device stimulation. However, it should be noted that we found no reduction in MEP amplitude elicited by PA TMS (Fig. 5A); by contrast, Tazoe and Perez (2017) did see a smaller MEP during a power grip than isolated finger abduction.

Previous work from this laboratory introduced the protocol of pairing auditory clicks with electrical muscle stimulation using a wearable device, and showed that it was capable of generating plastic changes in the LLSR (Foysal et al., 2016). The modality of paired stimuli, and their interstimulus interval, were originally chosen with the aim of targeting the RST. As the LLSR in biceps likely has a subcortical component (Kimura et al., 2006; Shemmell et al., 2009; Kurtzer, 2015), we speculated that plasticity had indeed been generated subcortically, possibly in the RST. In the present study, we have extended the evidence in support of this conclusion. Paired device stimulation increased the StartReact effect, decreased the suppression of MEPs by loud sounds, and increased MEPs elicited by an AP coil orientation. All of these changes are consistent with plasticity in subcortical pathways, including the RST. By contrast, MEPs generated by the PA coil orientation were unaltered, suggesting that plasticity did not occur in the CST. Non-invasive measures in humans are rarely completely unambiguous, but convergent results using multiple measurements build a more convincing case than any single finding. While we still cannot exclude a contribution from other circuits, we believe that the weight of evidence points toward changes in subcortical circuits, particularly the RST, following paired device stimulation.

An important question is why repeated convergent activation of auditory/vestibular and afferent inputs at the brainstem should alter a wide range of subcortical response measures, many of which are likely to rely on synapses in the corticoreticulospinal pathway, rather than those carrying sensory inputs to the brainstem. One possibility is that wearable device stimulation not only strengthened specific synapses related to the paired inputs, but also elevated brainstem excitability leading to a general increase in RST output. We have previously reported a similar situation during recovery from CST damage, where there are changes not only in selected RST connections to motoneurons (Zaaimi et al., 2012) but also increases in single cell activity within the reticular formation itself (Zaaimi et al., 2018).

\section{Potential for rehabilitation}

Stimulus rate is an important parameter in protocols which induce plasticity, and the direction of effects (potentiation vs suppression) can even be altered by changing rate (Pitcher et al., 2003). Importantly, here we extended our previous work (Foysal et al., 2016) by testing a higher frequency of paired stimulation. This did not significantly increase the size of plastic changes, indicating that the original frequency was at or above the optimal level. Anecdotally, some subjects found the higher stimulus rate irritating, whereas the lower rate was well tolerated. The similar results are thus encouraging, as they suggest that there is no benefit to using the more unpleasant stimulation. In our recent clinical trial of this device in stroke patients over a four-week period (Choudhury et al., 2020), patients who chose to wear the device for longer each day showed greater functional gains. This suggests that greater plastic changes can be generated by longer periods of paired stimulation. In the present study subjects always wore the device for $6 \mathrm{~h}$; further work is needed to define what stimulation duration is optimal.

Following extensive CST damage such as after stroke or spinal cord injury, the RST may be the only major descending motor pathway remaining and plays an important role in recovery (Baker, 2011; Zaaimi et al., 2012, 2018). Prior studies usually attempted to boost functional recovery using neurostimulation targeted cortical changes, and often met with disappointing results (Rothwell, 2016). The ability to manipulate plasticity in a subcortical target may be capable of achieving gains not accessible by other means.

\section{References}

Arai N, Okabe S, Furubayashi T, Terao Y, Yuasa K, Ugawa Y (2005) Comparison between short train, monophasic and biphasic repetitive transcranial magnetic stimulation (rTMS) of the human motor cortex. Clin Neurophysiol 116:605-613.

Baker SN (2011) The primate reticulospinal tract, hand function and functional recovery. J Physiol 589:5603-5612.

Baker SN, Perez MA (2017) Reticulospinal contributions to gross hand function after human spinal cord injury. J Neurosci 37:9778-9784.

Barrionuevo G, Brown TH (1983) Associative long-term potentiation in hippocampal slices. Proc Natl Acad Sci USA 80:7347-7351.

Benjamini Y, Hochberg Y (1995) Controlling the false discovery rate: a practical and powerful approach to multiple testing. J R Stat Soc Series B Stat Methodol 57:289-300.

Carlsen AN, Chua R, Inglis JT, Sanderson DJ, Franks IM (2004) Can prepared responses be stored subcortically? Exp Brain Res 159:301-309.

Cheeran B, Talelli P, Mori F, Koch G, Suppa A, Edwards M, Houlden H, Bhatia K, Greenwood R, Rothwell JC (2008) A common polymorphism in the brain-derived neurotrophic factor gene (BDNF) modulates human cortical plasticity and the response to rTMS. J Physiol 586:5717-5725.

Choudhury S, Shobhana A, Singh R, Sen D, Anand SS, Shubham S, Baker MR, Kumar H, Baker SN (2019) The relationship between enhanced reticulospinal outflow and upper limb function in chronic stroke patients. Neurorehabil Neural Repair 33:375-383.

Choudhury S, Singh R, Shobhana A, Sen D, Anand SS, Shubham S, Gangopadhyay S, Baker MR, Kumar H, Baker SN (2020) A novel wearable device for motor recovery of hand function in chronic stroke survivors. Neurorehabil Neural Repair 34:600-608.

Cirillo J, Perez MA (2015) Subcortical contribution to late TMS-induced Iwaves in intact humans. Front Integr Neurosci 9:38.

Cirillo J, Calabro FJ, Perez MA (2016) Impaired organization of paired-pulse TMS-induced I-waves after human spinal cord injury. Cereb Cortex 26:2167-2177.

Darling WG, Ge J, Stilwell-Morecraft KS, Rotella DL, Pizzimenti MA, Morecraft RJ (2018) Hand motor recovery following extensive frontoparietal cortical injury is accompanied by upregulated corticoreticular projections in monkey. J Neurosci 38:6323-6339. 
Davidson AG, Buford JA (2004) Motor outputs from the primate reticular formation to shoulder muscles as revealed by stimulus-triggered averaging. J Neurophysiol 92:83-95.

Davidson AG, Buford JA (2006) Bilateral actions of the reticulospinal tract on arm and shoulder muscles in the monkey: stimulus triggered averaging. Exp Brain Res 173:25-39.

Davidson AG, Schieber MH, Buford JA (2007) Bilateral spike-triggered average effects in arm and shoulder muscles from the monkey pontomedullary reticular formation. J Neurosci 27:8053-8058.

Davis M, Gendelman DS, Tischler MD, Gendelman PM (1982) A primary acoustic startle circuit: lesion and stimulation studies. J Neurosci 2:791805.

Day BL, Dressler D, Maertens de Noordhout A, Marsden CD, Nakashima K, Rothwell JC, Thompson PD (1989) Electric and magnetic stimulation of human motor cortex: surface EMG and single motor unit responses. J Physiol 412:449-473.

Dean LR, Baker SN (2017) Fractionation of muscle activity in rapid responses to startling cues. J Neurophysiol 117:1713-1719.

Delwaide PJ, Schepens B (1995) Auditory startle (audio-spinal) reaction in normal man: EMG responses and $\mathrm{H}$ reflex changes in antagonistic lower limb muscles. Electroencephalogr Clin Neurophysiol 97:416-423.

Di Lazzaro V, Oliviero A, Saturno E, Pilato F, Insola A, Mazzone P, Profice P, Tonali P, Rothwell JC (2001) The effect on corticospinal volleys of reversing the direction of current induced in the motor cortex by transcranial magnetic stimulation. Exp Brain Res 138:268-273.

Federico P, Perez MA (2017) Distinct corticocortical contributions to human precision and power grip. Cereb Cortex 27:5070-5082.

Fisher KM, Zaaimi B, Baker SN (2012) Reticular formation responses to magnetic brain stimulation of primary motor cortex. J Physiol 590:40454060.

Fisher KM, Jillani NE, Oluoch GO, Baker SN (2015) Blocking central pathways in the primate motor system using high-frequency sinusoidal current. J Neurophysiol 113:1670-1680.

Fisher KM, Zaaimi B, Edgley SA, Baker SN (2021) Extensive cortical convergence to primate reticulospinal pathways. J Neurosci, in press.

Foysal KMR, Baker SN (2019) A Hierarchy of corticospinal plasticity in human hand and forearm muscles. J Physiol 597:2729-2739.

Foysal KHMR, De Carvalho F, Baker SN (2016) Spike-timing dependent plasticity in the long latency stretch reflex following paired stimulation from a wearable electronic device. J Neurosci 36:10823-10830.

Fregosi M, Contestabile A, Hamadjida A, Rouiller EM (2017) Corticobulbar projections from distinct motor cortical areas to the reticular formation in macaque monkeys. Eur J Neurosci 45:1379-1395.

Furubayashi T, Ugawa Y, Terao Y, Hanajima R, Sakai K, Machii K, Mochizuki H, Shiio Y, Uesugi H, Enomoto H, Kanazawa I (2000) The human hand motor area is transiently suppressed by an unexpected auditory stimulus. Clin Neurophysiol 111:178-183.

Hammond GR (1973) Lesions of pontine and medullary reticular formation and prestimulus inhibition of the acoustic startle reaction in rats. Physiol Behav 10:239-243.

Honeycutt CF, Perreault EJ (2012) Planning of ballistic movement following stroke: insights from the startle reflex. PLoS One 7:e43097.

Honeycutt CF, Kharouta M, Perreault EJ (2013) Evidence for reticulospinal contributions to coordinated finger movements in humans. J Neurophysiol 110:1476-1483.

Irvine DR, Jackson GD (1983) Auditory input to neurons in mesencephalic and rostral pontine reticular formation: an electrophysiological and horseradish peroxidase study in the cat. J Neurophysiol 49:1319-1333.

Jankowska E, Padel Y, Tanaka R (1975) The mode of activation of pyramidal tract cells by intracortical stimuli. J Physiol 249:617-636.

Kimura T, Haggard P, Gomi H (2006) Transcranial magnetic stimulation over sensorimotor cortex disrupts anticipatory reflex gain modulation for skilled action. J Neurosci 26:9272-9281.

Kühn AA, Sharott A, Trottenberg T, Kupsch A, Brown P (2004) Motor cortex inhibition induced by acoustic stimulation. Exp Brain Res 158:120124.

Kurtzer IL (2015) Long-latency reflexes account for limb biomechanics through several supraspinal pathways. Front Integr Neurosc 8:99.

Ladpli R, Brodal A (1968) Experimental studies of commissural and reticular formation projections from the vestibular nuclei in the cat. Brain Res 8:65-96.
Leiras R, Velo P, Martín-Cora F, Canedo A (2010) Processing afferent proprioceptive information at the main cuneate nucleus of anesthetized cats. J Neurosci 30:15383-15399.

Leitner DS, Powers AS, Hoffman HS (1980) The neural substrate of the startle response. Physiol Behav 25:291-297.

Long J, Federico P, Perez MA (2017) A novel cortical target to enhance hand motor output in humans with spinal cord injury. Brain 140:1619-1632.

Markram H, Lübke J, Frotscher M, Sakmann B (1997) Regulation of synaptic efficacy by coincidence of postsynaptic APs and EPSPs. Science 275:213215.

McNeil CJ, Butler JE, Taylor JL, Gandevia SC (2013) Testing the excitability of human motoneurons. Front Hum Neurosci 7:152.

Nakajima T, Tazoe T, Sakamoto M, Endoh T, Shibuya S, Elias LA, Mezzarane RA, Komiyama T, Ohki Y (2017) Reassessment of nonmonosynaptic excitation from the motor cortex to motoneurons in single motor units of the human biceps brachii. Front Hum Neurosci 11:19.

Nakashima K, Wang Y, Shimoda M, Shimoyama R, Yokoyama Y, Takahashi K (1994) Auditory effects on the motor responses after magnetic cortical stimulation and on the H-reflexes in patients with Parkinson's disease. J Neurol Sci 122:15-19.

Nathan PW, Smith MC (1955) Long descending tracts in man. I. Review of present knowledge. Brain 78:248-303.

Ni Z, Charab S, Gunraj C, Nelson AJ, Udupa K, Yeh IJ, Chen R (2011) Transcranial magnetic stimulation in different current directions activates separate cortical circuits. J Neurophysiol 105:749-756.

Nonnekes J, Oude Nijhuis LB, de Niet M, de Bot ST, Pasman JW, van de Warrenburg BP, Bloem BR, Weerdesteyn V, Geurts AC (2014) StartReact restores reaction time in HSP: evidence for subcortical release of a motor program. J Neurosci 34:275-281.

Onodera S, Hicks TP (2010) Carbocyanine dye usage in demarcating boundaries of the aged human red nucleus. PLoS One 5:e14430.

Pare D, Smith Y, Parent A, Steriade M (1988) Projections of brainstem core cholinergic and non-cholinergic neurons of cat to intralaminar and reticular thalamic nuclei. Neuroscience 25:69-86.

Patton HD, Amassian VE (1954) Single and multiple-unit analysis of cortical stage of pyramidal tract activation. J Neurophysiol 17:345-363.

Petersen NT, Taylor JL, Gandevia SC (2002) The effect of electrical stimulation of the corticospinal tract on motor units of the human biceps brachii. J Physiol 544:277-284.

Peterson BW, Abzug C (1975) Properties of projections from vestibular nuclei to medial reticular formation in the cat. J Neurophysiol 38:1421-1435.

Peterson BW, Maunz RA, Pitts NG, Mackel RG (1975) Patterns of projection and braching of reticulospinal neurons. Exp Brain Res 23:333-351.

Pitcher JB, Ridding MC, Miles TS (2003) Frequency-dependent, bi-directional plasticity in motor cortex of human adults. Clin Neurophysiol 114:1265-1271.

Ridding MC, Uy J (2003) Changes in motor cortical excitability induced by paired associative stimulation. Clin Neurophysiol 114:1437-1444.

Riddle CN, Edgley SA, Baker SN (2009) Direct and indirect connections with upper limb motoneurons from the primate reticulospinal tract. J Neurosci 29:4993-4999.

Rosengren SM, Welgampola MS, Colebatch JG (2010) Vestibular evoked myogenic potentials: past, present and future. Clin Neurophysiol 121:636-651.

Rosenthal J, Waller HJ, Amassian VE (1967) An analysis of the activation of motor cortical neurons by surface stimulation. J Neurophysiol 30:844858.

Rossignol S, Jones GM (1976) Audio-spinal influence in man studied by the $\mathrm{H}$-reflex and its possible role on rhythmic movements synchronized to sound. Electroencephalogr Clin Neurophysiol 41:83-92.

Rothwell JC (2006) The startle reflex, voluntary movement, and the reticulospinal tract. Suppl Clin Neurophysiol 58:223-231.

Rothwell JC (2016) Can motor recovery in stroke be improved by non-invasive brain stimulation? Adv Exp Med Biol 957:313-323.

Rothwell JC, Thompson PD, Day BL, Boyd S, Marsden CD (1991) Stimulation of the human motor cortex through the scalp. Exp Physiol 76:159-200.

Rothwell JC, Hallett M, Berardelli A, Eisen A, Rossini P, Paulus W (1999) Magnetic stimulation: motor evoked potentials. The International Federation of Clinical Neurophysiology. Electroencephalogr Clin Neurophysiol Suppl 52:97-103. 
Rudell AP, Eberle LP (1985) Acoustic facilitation of the Hoffmann reflex. Exp Neurol 89:592-602.

Sakai K, Ugawa Y, Terao Y, Hanajima R, Furubayashi T, Kanazawa I (1997) Preferential activation of different I waves by transcranial magnetic stimulation with a figure-of-eight-shaped coil. Exp Brain Res 113:24-32.

Shemmell J, An JH, Perreault EJ (2009) The differential role of motor cortex in stretch reflex modulation induced by changes in environmental mechanics and verbal instruction. J Neurosci 29:13255-13263.

Smith V, Maslovat D, Carlsen AN (2019) StartReact effects are dependent on engagement of startle reflex circuits: support for a subcortically mediated initiation pathway. J Neurophysiol 122:2541-2547.

Stefan K, Kunesch E, Cohen LG, Benecke R, Classen J (2000) Induction of plasticity in the human motor cortex by paired associative stimulation. Brain 123:572-584.

Steriade M, Pare D, Parent A, Smith Y (1988) Projections of cholinergic and non-cholinergic neurons of the brainstem core to relay and associational thalamic nuclei in the cat and macaque monkey. Neuroscience 25:47-67.

Stoney SD Jr, Thompson WD, Asanuma H (1968) Excitation of pyramidal tract cells by intracortical microstimulation: effective extent of stimulating current. J Neurophysiol 31:659-669.

Tazoe T, Perez MA (2017) Cortical and reticular contributions to human precision and power grip. J Physiol 595:2715-2730.
The Stationery Office (2005) Control of noise at work regulations. Available at https://www.legislation.gov.uk/uksi/2005/1643/contents/made.

Ugawa Y, Rothwell JC, Day BL, Thompson PD, Marsden CD (1991) Percutaneous electrical stimulation of corticospinal pathways at the level of the pyramidal decussation in humans. Ann Neurol 29:418-427.

Valls-Solé J, Rothwell JC, Goulart F, Cossu G, Muñoz E (1999) Patterned ballistic movements triggered by a startle in healthy humans. J Physiol 516:931-938

Werhahn KJ, Fong JKY, Meyer BU, Priori A, Rothwell JC, Day BL, Thompson PD (1994) The effect of magnetic coil orientation on the latency of surface EMG and single motor unit responses in the first dorsal interosseous muscle. Electroen Clin Neuro 93:138-146.

Wiethoff S, Hamada M, Rothwell JC (2014) Variability in response to transcranial direct current stimulation of the motor cortex. Brain Stimul 7:468-475.

Zaaimi B, Edgley SA, Soteropoulos DS, Baker SN (2012) Changes in descending motor pathway connectivity after corticospinal tract lesion in macaque monkey. Brain 135:2277-2289.

Zaaimi B, Soteropoulos DS, Fisher KM, Riddle CN, Baker SN (2018) Classification of neurons in the primate reticular formation and changes after recovery from pyramidal tract lesion. J Neurosci 38:6190-6206.

Ziemann U, Rothwell JC (2000) I-waves in motor cortex. J Clin Neurophysiol 17:397-405. 